\title{
Onto4LA: uma ontologia para integração de dados educacionais
}

\section{Luiz Carlos Carchedi ${ }^{1}$, Eduardo Barrére ${ }^{1}$, Fabrício Martins Mendonça ${ }^{1}$, Jairo Francisco de Souza ${ }^{1}$}

\author{
${ }^{1}$ Pós-graduação em Ciência da Computação \\ Universidade Federal de Juiz de Fora (UFJF) - MG - Brasil \\ \{carchedi, eduardo.barrere, fabricio.mendonca, jairo.souza\}@ice.ufjf.br
}

\begin{abstract}
Nowadays there are diferent systems to support teaching and learning, each one has their own objectives and using specifics methods to collect educational data. To conciliate data from different sources is a problem of the Learning Analytics (LA) area. To help in this task, this article presents the initial version of Ontology For Learning Analytics (Onto4LA), created with the objective of facilitating the integration of educational data. The main contribuition of the proposed ontology is make possible the integration of different concepts presents on the frameworks of Learning Analytics.
\end{abstract}

Resumo. Atualmente existem diferentes sistemas para apoio ao ensino e aprendizagem, cada um deles com seus próprios objetivos e utilizando métodos específicos para a coleta de dados educacionais. A integração desses dados oriundos de diferentes fontes é um dos problemas da área de Learning Analytics (LA). Para auxiliar nesta tarefa, este artigo apresenta a versão inicial da Ontology For Learning Analytics (Onto4LA), criada com o objetivo de facilitar a integração de dados educacionais. A principal contribuição da ontologia é tornar possível a integração de diferentes conceitos presentes nos frameworks de Learning Analytics.

\section{Introdução}

A educação a distância é uma tecnologia em ascensão e um dos motivos que justificam sua grande quantidade de usuários é a flexibilidade que essa modalidade oferece [Lazzarotti Filho et al. 2015], como horário e local de acesso. Isso faz com que os Ambientes Virtuais de Aprendizagem (AVA) sejam utilizados para a gestão do ensino a distância. O crescente uso dos AVA's resulta em uma quantidade cada vez maior de dados gerados pelos mesmos [Silva 2016].

Atualmente existem diferentes AVA's, cada um deles com seus próprios objetivos e utilizando métodos específicos para a coleta de dados educacionais. Utilizar dados de diversas fontes, com formatos e objetivos distintos, é uma tarefa que exige muito esforço manual e análise do usuário. Assim, torna-se necessária a busca por soluções que permitam organizar os dados vindos de diferentes ferramentas e processá-los adequadamente.

Para auxiliar na integração desses dados, este trabalho apresenta uma ontologia criada de forma a abranger os mais variados tipos de informação geradas. Para isso, foram analisadas as diversas propostas de dashboards existentes na literatura e, a partir 
VII Congresso Brasileiro de Informática na Educação (CBIE 2018)

Anais dos Workshops do VII Congresso Brasileiro de Informática na Educação (WCBIE 2018)

da detecção dos dados necessários para cada ferramenta, foi definida uma ontologia com conceitos em alto nível de forma a representar esses diferentes dados.

O trabalho está organizado nas seguintes seções: a Seção 2 descreve o problema de integração dos dados e o papel de Learning Analytics e ontologias nesse contexto; na Seção 3 são apresentados alguns frameworks relevantes para Learning Analytics; a Seção 4 apresenta a versão inicial da ontologia Onto4LA e, por fim, a Seção 5 apresenta as conclusões dos autores e trabalhos futuros.

\section{O problema de integração de dados: papel de Learning Analytics e das ontologias}

A integração e análise de dados produzidos por diferentes sistemas de informação é um dos problemas mais recorrentes para a área de Computação e continua a ser um grande desafio, especialmente pelo grande volume de dados produzidos atualmente. Com o propósito de buscar soluções para o problema de integração e análise de dados, foram desenvolvidos, recentemente, recursos que tratam dessa questão. Neste artigo, aborda-se dois temas voltados para esse problema: a área de Learning Analytics e o uso de ontologias para interoperabilidade semântica.

A área de Learning Analytics (LA) é uma ciência voltada para a conversão de dados educacionais em informações que permitam a realização de ações para apoiar a melhoria no processo de aprendizagem [Chatti et al. 2012]. LA é um campo de estudos emergente, onde ferramentas analíticas podem ser usadas para melhorar o processo de aprendizagem e a área da educação [Elias 2011]. Para isso, a área de LA necessita realizar a medição, coleta e análise de dados educacionais das atividades de alunos e professores para identificar padrões de comportamento e entender melhor o processo de ensinoaprendizagem [Chatti et al. 2012]. O processo geral de LA tem início na coleta de dados, seguida por um processo de mineração desses dados, e a partir da análise gráfica dos dados, os avaliadores devem ser capazes de interpretar mais rapidamente as informações visualizadas e decidir se os objetivos foram alcançados [Dyckhoff et al. 2012].

No contexto de LA, um grande volume de dados não uniformizado e distribuído em diferentes bases de dados dificulta a realização de análises mais significativas. Alguns autores têm proposto soluções para tratar o problema de integração. Por exemplo, o trabalho proposto em [Savitraz 2010] tem como objetivo uma abordagem de integração dos dados educacionais produzidos pelo INEP, uma vez que não existe uma estrutura padrão para armazenar e distribuir tais dados, dificultando o acesso e a integração destes com outros sistemas. Nesse trabalho, o principal problema relatado é a dificuldade em padronizar os dados que são disponibilizados em formato textual e não possuem metadados que permitam que os mesmos sejam manipulados segundo a sua semântica. $\mathrm{O}$ autor propõe a criação de um mecanismo para armazenar os dados e os metadados das avaliações realizadas no sistema, para que dessa forma, seja possível uma abordagem que realize a busca de dados também pela parte semântica.

Ontologias são artefatos de representação da informação muito úteis para integração de dados e para garantir interoperabilidade semântica das informações que ela representa. A construção de representações como ontologias tem por objetivo desenvolver meios para que as máquinas possam servir aos humanos de maneira mais eficiente [Berners-Lee et al. 2001], de tal forma que as buscas dos usuários por uma dada 
VII Congresso Brasileiro de Informática na Educação (CBIE 2018)

Anais dos Workshops do VII Congresso Brasileiro de Informática na Educação (WCBIE 2018)

informação recuperem informações integradas, as mais precisas possíveis e sem ambiguidades, além da possibilidade de inferência/dedução de informações por parte das máquinas. Para atingir tal objetivo, as ontologias fornecem um conjunto de definições de conceitos e o relacionamento entre eles, expressos através de formalismos lógicos, visando um entendimento universal de dados heterogêneos [W3C 2017].

O conceito de interoperabilidade emerge da necessidade dos dados, primeiramente produzidos de forma independente e acarretando em heterogeneidade, terem uma estrutura uniforme e integrada, permitindo o compartilhamento. Tratar a interoperabilidade é uma das principais metas atuais do consórcio W3C, o qual vem se dedicando no desenvolvimento de padrões para avançar rumo a excelência em termos de interoperabilidade. Neste contexto, as ontologias cumprem um papel importante para tratar a interoperabilidade semântica, aumentando assim o potencial informacional entre os sistemas. Em alguns casos, projetos governamentais, como visto em [Farinelli et al. 2014] têm surgido para auxiliar no uso de informações oriundas de diferentes fontes.

A partir do uso de ontologias, torna-se possível a integração de dados educacionais originados a partir frameworks diferentes, utilizados no contexto de LA. A partir dessa integração, tem-se a interoperabilidade entre os dados, não existindo mais dependência da ferramenta que o forneceu.

\section{Frameworks para Learning Analytics}

Os frameworks são desenvolvidos para o suporte tanto de alunos quanto de professores de maneira cooperativa, tanto para aprendizado em conjunto quanto pra aprendizado individual [Einhardt et al. 2016]. Entre os frameworks voltados para a área de LA, existem trabalhos a respeito dos que são apenas conceituais e também trabalhos relacionados a frameworks que foram implementados.

Em [Dawson and Siemens 2014], por exemplo, é proposto um framework conceitual para a análise da aprendizagem. O objetivo da proposta é auxiliar na medição da aprendizagem multidisciplinar e permitir uma perspectiva institucional do progresso educacional. Em [Sedrakyan et al. 2018] é proposto outro modelo conceitual focado na relação existente entre a parte visual do dashboard e as informações a respeito do comportamento e desenvolvimento da turma e ainda como o feedback dos estudantes ajuda em um melhor direcionamento do desenvolvimento do curso. Em ambos os casos, o foco dos trabalhos é um conceito de framework que atenda as demandas de aprendizagem e consiga fornecer as informações referentes ao progresso do aluno durante o curso.

Em [Verbert et al. 2013] é apresentado o StepUP, outro framework conceitual para a visualização de características no processo de aprendizagem através dos AVA's. Nesse trabalho o framework é comparado com mais 15 dashboards em termos de público alvo (alunos/professores), tempo de uso, interação social, ferramentas disponíveis, material produzido e exercícios disponibilizados. De maneira semelhante, em [Einhardt et al. 2016] é descrito o Moodle Analytics Dashboard (MAD), uma ferramenta desenvolvida para permitir a visualização de atividades desenvolvidas por alunos e professores no ambiente Moodle, ajudando assim os professores a seguir melhor caminho para o ensino e o processo de aprendizagem. Em seguida, esse framework é comparado com outros 8 frameworks em relação a: público alvo, AVA's utilizados, disponibilidade, gráficos para apresentação de resultados e métricas utilizadas. 
VII Congresso Brasileiro de Informática na Educação (CBIE 2018)

Anais dos Workshops do VII Congresso Brasileiro de Informática na Educação (WCBIE 2018)

Em relação aos frameworks que foram implementados, temos o presente em [Fernández-Gallego et al. 2013], o qual apresenta uma análise da aprendizagem fundamentada na descoberta de fluxos de aprendizado. O núcleo do framework dos autores é uma plataforma baseada em Opensim, que tem a capacidade de monitorar e registrar os eventos gerados por alunos e professores. Com base nesses logs de eventos, os algoritmos de mineração de processo extraem automaticamente o fluxo real de aprendizado do curso, permitindo que os professores introduzam mudanças no mesmo para que seja viável atingir o aprendizado inicialmente proposto.

Ao comparar os frameworks acima, é possível identificar as características e objetivos de cada um, além de elencar as informações que os mesmos processam. Assim, foi feito um levantamento dos tipos de informação mais comuns presentes nestas soluções a fim de definir um modeo de dados que permita padronizá-las e disponibilizá-las.

\section{Construção da ontologia Onto4LA para integração de dados}

Nesta seção é apresentado o processo de construção da ontologia Onto4LA. Em seguida, é apresentado parte do conteúdo desta ontologia, através do seu conjunto de classes e alguns exemplos de modelagem de eventos com uso destas classes.

\subsection{Prospecção de conceitos para a ontologia}

Para definir a ontologia abrangendo o máximo de conceitos presentes nos frameworks de LA da literatura, foram analisados diferentes trabalhos. Foram selecionados os frameworks que tem foco voltado para a interação social (comunicação entre os usuários), avaliação do aluno, acesso à ferramenta, tempo de uso, material produzido e exercícios disponibilizados, uma vez que são as propostas mais presentes na literatura. Após o levantamento da literatura, através de artigos que trazem análises de frameworks [Verbert et al. 2014, Einhardt et al. 2016], os seguintes foram analisados: Agent ADVisor, Agent Inspector, Classroom View, Course Signals, CourseVis, KEATS Analytics, LOCO-Analyst, LOOP, Moodle Dashboard, SAM, SmartKlass, SNAPP, StepUP, TUT Circle Dashboard.

Para cada framework, foram analisadas as suas características e os tipos de dados que fornecem. Feito isso, foram levantadas as informações disponibilizadas nessas ferramentas e, em seguida, gerado um documento descrevendo esses tipos de informação de maneira mais abstrata. No total, foram descritos dezessete tipos de informação, pelos quais todas as informações fornecidas por todos os frameworks são geradas:

- Obter o perfil de um aluno

- Troca de mensagens entre dois agentes

- Envio de mensagens de um grupo para um agente

- Envio de mensagens de um agente para um grupo

- Logs de mensagens trocadas

- Interações do Aluno com a Ferramenta

- Interações sociais
- Histórico Acadêmico

- Histórico Uso da ferramenta

- Preferências do aluno

- Avaliação de um aluno

- Avaliação de um grupo de alunos

- Tempo de utilização da ferramenta

- Material Acessado

- Resultado de Testes

- Material disponível

- Material desenvolvido na Ferramenta 
Com os tipos de informação acima, foi adotada a estratégia de construção bottomup para criar classes do domínio educacional que pudessem integrar todos esses tipos levantados. No uso de tal estratégia, foram levantadas quais informações precisam ser fornecidas por cada framework pesquisado, tais informações foram agregadas e deram origem às classes da ontologia, que conseguem integrar e fornecer as informações educacionais necessárias para este estudo. As próximas seções descrevem o conteúdo da ontologia Onto4LA e apresenta alguns de seus exemplos de uso.

\subsection{Reúso de conceitos de ontologias de alto nível}

Esta subseção do artigo apresenta a etapa de reutilização usada para construção da ontologia proposta. Inicialmente, foi realizada uma pesquisa sobre ontologias de alto nível [Guarino 1998] que pudessem ser utilizadas como ponto de partida para o desenvolvimento ontológico aqui proposto. Geralmente, as ontologias de alto nível são usadas como base ou ponto de partida para a construção de ontologias sobre domínios específicos, tal que essa é uma prática científica bem aceita na literatura da área. Para a modelagem dessa ontologia, foram utilizados conceitos presentes nas ontologias descritas abaixo.

A ontologia Simple Event Model (SEM) ${ }^{1}$ [van Hage et al. 2011] é uma ontologia cujo objetivo é a modelagem de eventos. Os eventos tornaram-se elementos centrais na representação de dados em muitos domínios. A ontologia é criada para modelar eventos nesses vários domínios, sem fazer suposições sobre os vocabulários específicos de domínio usados. Como nessa ontologia temos os conceitos de evento, fundamental para a ontologia desenvolvida, ela foi utilizada como fundamento para a nova ontologia.

\section{A ontologia Simple Knowledge Organization System $(\mathrm{SKOS})^{2}$} [Miles and Bechhofer 2009] é voltada para um compartilhar e vincular sistemas de organização do conhecimento pela Web. Possui um modelo de dados que possibilita desenvolver e compartilhar novos sistemas de organização do conhecimento para a Web Semântica. Ela pode ser usada sozinha ou em combinação com linguagens formais de representação de conhecimento. Na ontologia proposta há o conceito de local, e como a ontologia SKOS apresenta o conceito de lugar real e virtual, ela foi reutilizada.

A ontologia FOAF $^{3}$ [Finin et al. 2005] é usada para representar pessoas e relações entre indivíduos online, fundamentada na ideia da web semântica. A ontologia FOAF permite descrever uma rede social sem que seja necessária a existência de uma base centralizada. Como a ontologia proposta tem classes que representam pessoas, essa ontologia foi mais adequada.

A Time Ontology ${ }^{4}$ foi criada para descrever os recursos temporais nos sistemas virtuais. Fornece meios de expressar instantes, intervalos, durações e posição temporal, incluindo data e hora [W3C 2017]. Na ontologia proposta, a ideia de tempo é importante e fundamental, dessa forma, a ontologia Time também foi usada como base.

\footnotetext{
${ }^{1}$ https://semanticweb.cs.vu.n1/2009/11/sem/

${ }^{2}$ https://www.w3.org/TR/2008/WD-skos-reference-20080829/skos.html

${ }^{3} \mathrm{http}: / /$ www.foaf-project.org/

${ }^{4}$ https://www.w3.org/TR/owl-time/
} 
VII Congresso Brasileiro de Informática na Educação (CBIE 2018)

Anais dos Workshops do VII Congresso Brasileiro de Informática na Educação (WCBIE 2018)

\subsection{Conteúdo da ontologia Onto4LA}

A ontologia Onto4LA pode ser caracterizada como uma ontologia de eventos. Uma ontologia de eventos define de maneira formal e explícita um modelo de classes de eventos que existem objetivamente; tais classes fornecem uma maneira natural de expressar relações complicadas entre pessoas, lugares, ações e objetos. Relacionamentos de eventos fornecem descrição e raciocínio mais sofisticados de conceitos centrados em eventos [Zhang et al. 2015]. Nesse sentido, a criação de uma ontologia de eventos é útil para resolver o problema de integração dos dados em diferentes padrões porque com ela é possível modelar os eventos que geram esses dados e fazer com que os dados sejam gerados de acordo com um padrão pré-estabelecido.

A Onto4LA também pode ser classificada como uma ontologia de domínio por representar conceitos de um domínio específico [Guarino 1998], neste caso, a educação. Sobre seu conteúdo ontológico, em sua versão inicial, a Onto4LA engloba um total de 27 classes, das quais 4 são reutilizadas da SEM Ontology, 2 da FOAF, 4 da SKOS e 3 da Time Ontology, e um total de 6 relações ontológicas (is_a, hasAgent, hasTime, hasPlace, produces, source). A ontologia foi criada de forma a permitir que conceitos como aluno, professor, etc, sejam contemplados. Esses conceitos, contudo, foram modelados utilizando outros nomes, os quais destacamos ao longo dessa seção.

Nessa ontologia, há classes que são a generalização de classes semelhantes, como, por exemplo, a classe Agent, com conceitos da ontologia FOAF, representa os participantes do sistema, tal como alunos, professores e outros; as subclasses de Thing representam o que é gerado ou mesmo o que é utilizado em cada um dos eventos. Nessa ontologia, as classes mais específicas foram determinadas para representar características necessárias para a especificação de um evento a ser descrito. A Figura 1 representa graficamente a ontologia. As classes reutilizadas de outras ontologias estão destacadas com cores distintas, conforme a legenda da figura. As setas de cor cinza representam relações do tipo is_a.

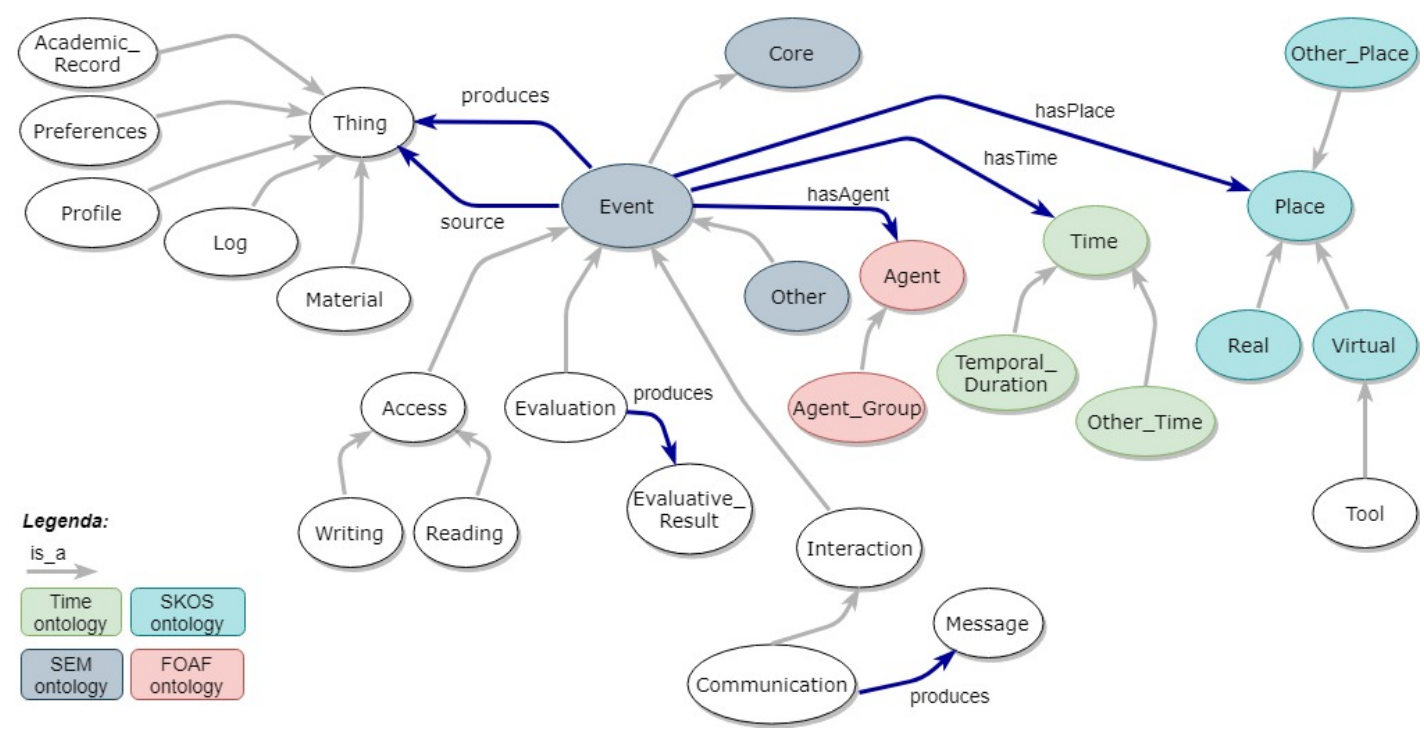

Figura 1. Visualização simplificada da Onto4LA

Faz-se necessário na construção de qualquer ontologia apresentar definições precisas e concisas para cada classe especificada. Nesta versão inicial da Onto4LA, as 
VII Congresso Brasileiro de Informática na Educação (CBIE 2018)

Anais dos Workshops do VII Congresso Brasileiro de Informática na Educação (WCBIE 2018)

\begin{tabular}{|ll|}
\hline Classe & Definição \\
\hline Academic_Record & $\begin{array}{l}\text { Registros acadêmicos são registros sobre as atividades do } \\
\text { aluno curso, seu histórico acadêmico ou mesmo seu desem- } \\
\text { penho durante o curso. }\end{array}$ \\
\hline Access & $\begin{array}{l}\text { Representa os acessos realizados à alguma ferramenta por al- } \\
\text { gum agente. }\end{array}$ \\
\hline Communication & $\begin{array}{l}\text { Representa eventos de comunicação, por exemplo, uma troca } \\
\text { de informação entre agents ou agents_groups. }\end{array}$ \\
\hline Evaluation & $\begin{array}{l}\text { Eventos avaliativos que ocorrem para medir a evolução de um } \\
\text { aluno no curso. }\end{array}$ \\
\hline Evaluative_Results & Representa resultados dos eventos de avaliação. \\
\hline Interaction & Evento de interação entre agentes com sistemas. \\
\hline Log & Registro de dados de um dado sistema \\
\hline Message & $\begin{array}{l}\text { Representa a informação enviada ou recebida por agents ou } \\
\text { agents_group }\end{array}$ \\
\hline Material & Produto produzido ou consumido durante um evento. \\
\hline Preferences & $\begin{array}{l}\text { Representa dados a respeito de um usuário baseados na sua } \\
\text { utilização do sistema. }\end{array}$ \\
\hline Profile & $\begin{array}{l}\text { O perfil de um agente é o conjunto de características que a } \\
\text { ferramenta pode fornecer a respeito de um usuário. }\end{array}$ \\
\hline Reading & $\begin{array}{l}\text { Evento que ocorre quando um usuário do sistema tem acesso a } \\
\text { algum material disponibilizado. }\end{array}$ \\
\hline Tool & $\begin{array}{l}\text { Representa um sistema ou uma ferramenta dentro de um sis- } \\
\text { tema. }\end{array}$ \\
\hline Writing & $\begin{array}{l}\text { A produção de material é um evento que acontece quando um } \\
\text { agent produz algum tipo de conteúdo. }\end{array}$ \\
\hline
\end{tabular}

Tabela 1. Descrição das classes da ontologia proposta

definições (Tabela 1) estão em linguagem natural. Na próxima versão, estas definições e demais propriedades das classes serão especificadas formalmente através da linguagem OWL. Para o propósito deste artigo, as definições em linguagem natural foram suficientes; ainda na Tabela 1, foram definidas as classes específicas da ontologia Onto4LA, as demais classes estão definidas nas respectivas ontologias de onde foram herdadas.

Com o objetivo de exemplificar o uso da Onto4LA, foram modelados alguns tipos de informações comuns aos frameworks descritos na seção 4.1. A Tabela 2 apresenta dois exemplos de modelagem de tipos de informação na forma de eventos. No exemplo 2.a estão os dados de comunicação de um professor com um grupo de alunos. No exemplo 2.b estão os dados do evento da avaliação de um aluno. 
VII Congresso Brasileiro de Informática na Educação (CBIE 2018)

Anais dos Workshops do VII Congresso Brasileiro de Informática na Educação (WCBIE 2018)

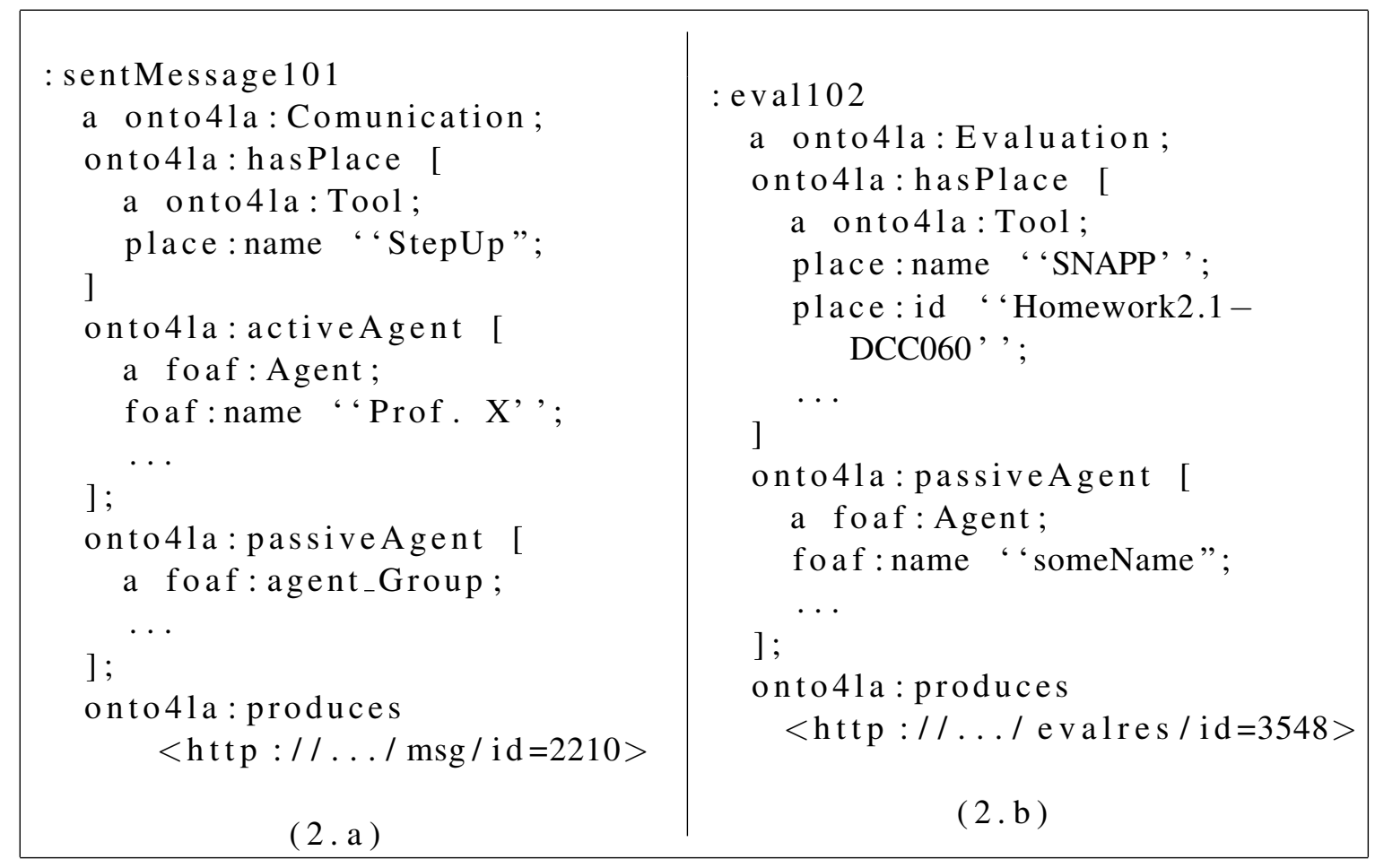

Tabela 2. Exemplos de uso da ontologia Onto4LA

A maioria dos frameworks analisados integram dados de interação entre usuários (alunos e professores), informações ou dados de processos avaliativos. No exemplo (2.a), a mensagem enviada por um professor para um grupo de alunos é modelada como uma instância (sentMessage 101) do evento Comunication. A mensagem foi enviada através da ferramenta StepUP. Nesse evento, o agente ativo é da classe Agent (o professor) e o agente passivo da classe Agent_Group (os alunos). Esse evento têm como resultado uma instância da classe Message previamente definida. Por sua vez, o exemplo (2.b) representa os valores de uma avaliação realizada na ferramenta SNAPP modelado como uma instância do evento de Evaluation. Os valores brutos da avaliação estão armazenados na instância evalres:3548, a qual está relacionada com a instância de evento eval102 através da relação onto4la:produces.

Assim como nesses exemplos, todas as demais situações possíveis nas ferramentas também podem ser modeladas como eventos na Onto4LA. Todos esses eventos sempre produzem informações que serão provenientes de alguma das classes definidas na ontologia. O foco dessa ontologia são os dados que são gerados ao final de cada evento, de maneira que o local do evento será uma das ferramentas (um local virtual), os agentes (passivo/ativo) podem ou não fazer parte de um determinado evento e os valores que se quer analisar posteriormente estarão acessíveis a partir da propriedade onto4la:produces. Uma vez definida a ontologia, suas classes, e quais dados essas classes precisam fornecer, é esperado que as ferramentas que se utilizarão da ontologia modelem seus dados nesse formato. Como o modelo de eventos é generalista, torna-se mais fácil representar novos tipos de informação e facilita a consulta e processamento desses dados neste formato. 
VII Congresso Brasileiro de Informática na Educação (CBIE 2018)

Anais dos Workshops do VII Congresso Brasileiro de Informática na Educação (WCBIE 2018)

\section{Conclusões}

O crescente uso de novas tecnologias da informação e comunicação no processo de educação a distância tem trazido facilidades nessa modalidade de ensino, bem como contribuído para seu crescimento em nosso país. O número de AVA's utilizados por diferentes instituições de ensino vem crescendo e produzindo um grande volume de dados.

Esse crescimento informacional no processo de ensino a distância traz benefícios para área, mas também amplia um problema bem comum para a área de Computação, que se refere à integração de dados de fontes heterogêneas, em geral sistemas de informação, que não se intercomunicam por não possuir um padrão de integração. Tratar a interoperabilidade é uma das principais metas atuais do consórcio W3C, o qual vem se dedicando no desenvolvimento de padrões para este fim.

Considerando esse cenário, o presente artigo apresentou uma solução a fim de colaborar com o problema citado, tendo como base a proposição da ontologia Onto4LA, desenvolvida para fornecer uma padronização de conceitos de Learning Analytics presentes nos frameworks aqui pesquisados.

Ao reutilizar classes de ontologias de alto nível e especificar classes e relações do domínio educacional, a Onto4LA mostrou ser bastante útil como uma ontologia para modelar todos os eventos que produzem informações nos frameworks pesquisados, como demonstrado nos exemplos da Tabela 2 da seção anterior. Um dos diferenciais da Onto4LA para outras ontologias do domínio da educação é que ela foi desenvolvida para englobar conceitos de uma área nova como Learning Analytics e possibilita formalizar os eventos modelados através de uma sintaxe simples e interpretável.

Como trabalhos futuros, a ontologia proposta, será adotada em um framework a ser desenvolvido para integração de dados educacionais de apoio a sistemas de Learning Analytics, dessa forma, será possível realizar testes de aceitação da ontologia. Tal framework auxiliará nas principais tarefas de integração e transformação de dados que atualmente estão distribuídos por diferentes ferramentas educacionais, sem um padrão. O framework irá também fornecer funções para processamento e visualização dos dados, de forma mais simplificada ao usuário, através de seus dashboards. Em paralelo com o desenvolvimento do framework citado, a ontologia Onto4LA será incrementada com formalismos lógicos para suas classes, relações e propriedades definidos em OWL.

\section{Referências}

Berners-Lee, T., Hendler, J., and Lassila, O. (2001). The semantic web. Scientific american, 284(5):34-43.

Chatti, M. A., Dyckhoff, A. L., Schroeder, U., and Thüs, H. (2012). A reference model for learning analytics. International Journal of Technology Enhanced Learning, 4(56):318-331.

Dawson, S. and Siemens, G. (2014). Analytics to literacies: The development of a learning analytics framework for multiliteracies assessment. The International Review of Research in Open and Distributed Learning, 15(4).

Dyckhoff, A. L., Zielke, D., Bültmann, M., Chatti, M. A., and Schroeder, U. (2012). Design and implementation of a learning analytics toolkit for teachers. Journal of Educational Technology \& Society, 15(3). 
VII Congresso Brasileiro de Informática na Educação (CBIE 2018)

Anais dos Workshops do VII Congresso Brasileiro de Informática na Educação (WCBIE 2018)

Einhardt, L., Tavares, T. A., and Cechinel, C. (2016). Moodle analytics dashboard: A learning analytics tool to visualize users interactions in moodle. In Learning Objects and Technology (LACLO), Latin American Conference on, pages 1-6. IEEE.

Elias, T. (2011). Learning analytics: Definitions, processes and potential. http://citeseerx.ist.psu.edu/viewdoc/summary?doi=10.1. 1.456 .7092 .

Farinelli, F., Melo, S., and Almeida, M. B. (2014). O papel das ontologias na interoperabilidade de sistemas de informação: reflexões na esfera governamental.

Fernández-Gallego, B., Lama, M., Vidal, J. C., and Mucientes, M. (2013). Learning analytics framework for educational virtual worlds. Procedia Computer Science, 25:443-447.

Finin, T., Ding, L., Zhou, L., and Joshi, A. (2005). Social networking on the semantic web. The Learning Organization, 12(5):418-435.

Guarino, N. (1998). Formal ontology in information systems: Proceedings of the first international conference (FOIS'98), June 6-8, Trento, Italy, volume 46. IOS press.

Lazzarotti Filho, A., Cruvinel, F., Silva, A. M., da Silva, M. Z., and de Almeida, G. C. F. (2015). A dinâmica, os principais problemas e as qualidades no desenvolvimento de um curso de licenciatura em educação física na modalidade a distância. Pensar a Prática, 18(3).

Miles, A. and Bechhofer, S. (2009). Skos simple knowledge organization system reference. $W 3 C$ recommendation, 18:W3C.

Savitraz, J. (2010). Uma abordagem de integração e exploração visual de dados educacionais na plataforma web-pide.

Sedrakyan, G., Malmberg, J., Verbert, K., Järvelä, S., and Kirschner, P. A. (2018). Linking learning behavior analytics and learning science concepts: designing a learning analytics dashboard for feedback to support learning regulation. Computers in Human Behavior.

Silva, CB; Oliveira, I. (2016). Learning analytics: Revisão da literatura e o estado da arte. http://www.abed.org.br/congresso2016/trabalhos/329.pdf.

van Hage, W. R., Malaisé, V., Segers, R., Hollink, L., and Schreiber, G. (2011). Design and use of the simple event model (sem). Web Semantics: Science, Services and Agents on the World Wide Web, 9(2):128 - 136. Provenance in the Semantic Web.

Verbert, K., Duval, E., Klerkx, J., Govaerts, S., and Santos, J. L. (2013). Learning analytics dashboard applications. American Behavioral Scientist, 57(10):1500-1509.

Verbert, K., Govaerts, S., Duval, E., Santos, J. L., Assche, F., Parra, G., and Klerkx, J. (2014). Learning dashboards: an overview and future research opportunities. Personal and Ubiquitous Computing, 18(6):1499-1514.

W3C (2017). Time ontology in owl. https://www.w3.org/TR/owl-time/.

Zhang, Y., Liu, W., Ding, N., Wang, X., and Tan, Y. (2015). An event ontology description framework based on skos. In IEEE 12th International Conference on Autonomic and Trusted Computing (UIC-ATC-ScalCom), pages 1774-1779. IEEE. 